\section{OPEN ACCESS}

Edited by:

Sandro M. Krieg,

Technische Universität München,

Germany

Reviewed by:

Sunit Das,

St. Michael's Hospital, Toronto,

Canada

Carsten Friedrich,

University of Rostock, Germany

*Correspondence:

Tanja Schneider

tan.schneider@uke.de

Specialty section:

This article was submitted to

Neuro-Oncology and Neurosurgical

Oncology,

a section of the journal

Frontiers in Neurology

Received: 27 March 2018

Accepted: 14 May 2018

Published: 30 May 2018

Citation:

Schneider T, Kemmling A, Schroeder J, Pantel K, Glatzel M, Schoen G, Mohme M, Fiehler J and GelliBen S (2018) Inverse Perfusion

Requirements of Supra- and

Infratentorial Brain Metastases

Formation. Front. Neurol. 9:391

doi: 10.3389/fneur.2018.00391

\title{
Inverse Perfusion Requirements of Supra- and Infratentorial Brain Metastases Formation
}

\begin{abstract}
Tanja Schneider ${ }^{1,2 *}$, André Kemmling ${ }^{3}$, Julian Schroeder ${ }^{4}$, Klaus Pantel $^{5}$, Markus Glatzel $^{6}$, Gerhard Schoen ${ }^{7}$, Malte Mohme ${ }^{8}$, Jens Fiehler ${ }^{1}$ and Susanne Gellißen ${ }^{1}$

${ }^{1}$ Department of Diagnostic and Interventional Neuroradiology, University Medical Center Hamburg-Eppendorf, Hamburg, Germany, ${ }^{2}$ Department of Radiology, Schoen Klinik Hamburg Eilbek, Hamburg, Germany, ${ }^{3}$ Department of Neuroradiology, University Medical Center Schleswig-Holstein, Luebeck, Germany, ${ }^{4}$ Department of Neurology, University Medical Center Hamburg-Eppendorf, Hamburg, Germany, ${ }^{5}$ Center for Experimental Medicine, Institute of Tumor Biology, University Medical Center Hamburg-Eppendorf, Hamburg, Germany, ${ }^{6}$ Center for Diagnostics, Institute of Neuropathology, University Medical Center Hamburg-Eppendorf, Hamburg, Germany, ${ }^{7}$ Department of Medical Biometry and Epidemiology, University Medical Center Hamburg-Eppendorf, Hamburg, Germany, ${ }^{8}$ Department of Neurosurgery, University Medical Center Hamburg-Eppendorf, Hamburg, Germany
\end{abstract}

Background and Aims: Vascular border zones and the gray-white matter junction are preferred sites for the development of brain metastases (BM), whereas microvascular lesions are known to be a protective factor. In this proof of concept study, we aim to study the relationship of blood perfusion and the spatial distribution of BM.

Materials and Methods: An average CT perfusion atlas of 107 healthy patients was created. Voxel-wise reference perfusion values were extracted from BM-negative and BM-positive regions in a second cohort of 100 untreated patients harboring 809 BM confirmed by MRI. A comparison of regional perfusion values was performed using the independent $t$-test.

Results: In contrast to supratentorial BM that develop preferably in areas with lower $\mathrm{CBV} / \mathrm{CBF}$ and longer MTT/TTP compared to the average regional perfusion $(p<0.001)$, infratentorial BM showed a higher CBV/CBF and shorter MTT/TTP $(p<0.001)$.

Conclusion: Our results imply differing pathophysiological mechanisms underlying supra- and infratentorial BM spreading. The inverse perfusion patterns may result from differences in vascular supply, hemodynamic requirements, and/or production of pro-angiogenic factors.

\footnotetext{
Keywords: blood-brain barrier, cerebral blood flows, hemodynamics, MR tomography, multidetector computed tomography, neoplasm metastases, neoplasms, perfusion imaging
}

\section{INTRODUCTION}

In principal, there are two main hypotheses on the development of brain metastases (BM). The "seed and soil" hypothesis states that metastatic cells favor a particular biochemical environment or tend to colonize selectively in suitable tissues (soils) due to cell surface and endothelium properties $(1,2)$. Furthermore, the "anatomical-mechanical" theory proposes that dissemination of metastases follows arterial vessel caliber and local blood flow (3).

Prior studies already demonstrated that the presence and the amount of small vessel ischemic disease are protective factors against the development of BM (4-7). Hwang et al. found a 
higher propensity of BM in vascular border zones and the graywhite matter junction (8). These findings suggest a relevant impact of brain perfusion on BM formation.

Here, we aim to study the relationship of the spatial distribution of the BM of 100 untreated patients and the underlying blood perfusion pattern. We hypothesize that all BM would preferably develop in areas where physiological perfusion is rather high compared to areas without any BM. We further hypothesize that central necrotic BM tend to colonize in areas with less perfusion.

\section{MATERIALS AND METHODS}

This single center, retrospective study was conducted in compliance with the local ethics committee (Ethik-Kommission der Aerztekammer Hamburg, WF-018/15) with a waiver of informed consent.

\section{CT Perfusion Atlas}

Cerebral blood flow in cancer patients has been shown to be altered due to paraneoplastic changes, chemotherapy administration, neoangiogenesis, and/or disruption of the bloodbrain barrier $(9,10)$. Therefore, we used pooled whole-brain CT perfusion datasets of a reference population. The perfusion atlas was generated from 107 patients who were triaged by CT perfusion for symptoms of transient ischemic attack but without evidence of infarction or any perfusion abnormality, symptoms on follow up, or vascular abnormality that was verified by MRI $24 \mathrm{~h}$ later (11). Quantitative perfusion maps were obtained for cerebral blood volume (CBV in $\mathrm{ml} / 100 \mathrm{~g}$ brain tissue), cerebral blood flow (CBF in $\mathrm{ml} / 100 \mathrm{~g}$ brain tissue/min), mean transit time (MTT in s), and time to peak (TTP in s). CT perfusion was performed on a Somatom Flash scanner (Siemens Healthcare, Erlangen, Germany) by applying the following scan parameters: $80 \mathrm{kV}, 200 \mathrm{mAs}$, collimation $2 \times 64 \times 0.6 \mathrm{~mm}, 5 \mathrm{~mm}$ slice

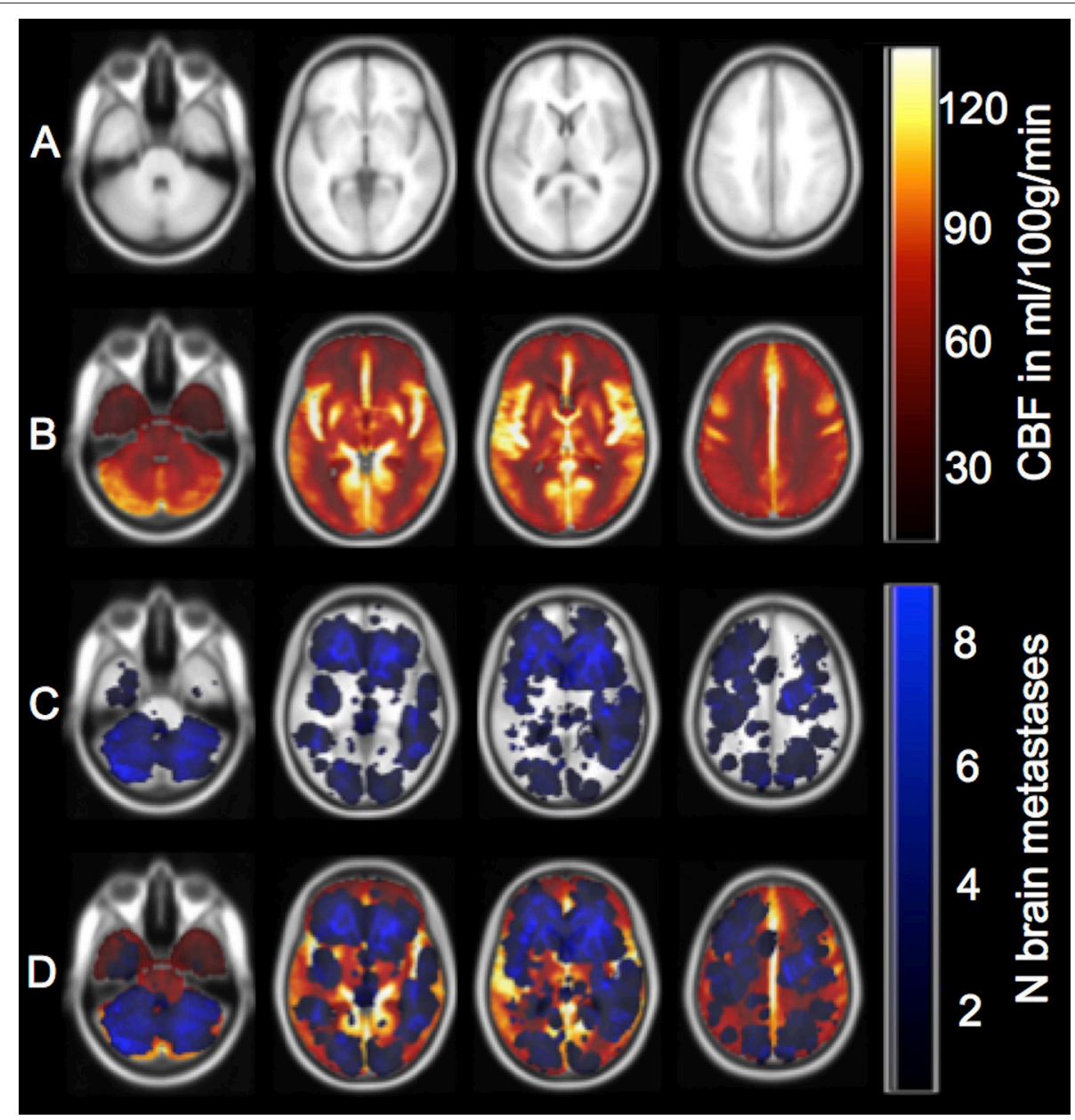

FIGURE 1 | Overview of perfusion calculation from caudal to cranial (1-4). (A) Example of the $1 \mathrm{~mm}$ MNI brain that served as a template for co-registration of the patient's contrast-enhanced T1-weighted images and the CT perfusion map. (B) Display of the cerebral blood flow (CBF) map (see color scale on the upper right; light yellow also corresponds to CBF values $\geq 120 \mathrm{ml} / 100 \mathrm{~g} / \mathrm{min}$ due to better visualization). Cerebral blood volume (CBV), mean transit time (MTT), and time to peak (TTP) maps are not depicted. (C) Cumulative brain metastases frequency map (see color scale on the lower right; light blue also corresponds to $\geq 8$ brain metastases due to better visualization). (D) Overlay of the metastases sum map, the perfusion map, and the MNI brain. CBF, CBV, MTT, and TTP values were extracted for each voxel-group with and without a BM. 
reconstruction, $1.5 \mathrm{~s}$ sampling rate for $45 \mathrm{~s}$, biphasic i.v. injection with $30 \mathrm{ml}$ of $400 \mathrm{mM} / \mathrm{ml}$ iodinated contrast medium followed by $30 \mathrm{ml} \mathrm{NaCl}$ chaser bolus with a flow rate of $4 \mathrm{ml} / \mathrm{s}$.

All perfusion raw data were processed in a central corelab on a workstation dedicated for perfusion analysis with motion correction and low band temporal noise removal (Syngo mmwp VE52A with VPCT-Neuro; Siemens Healthcare, Erlangen, Germany). Non-parenchymal voxels corresponding to bone, vasculature, calcification, and cerebrospinal fluid were automatically excluded by adaptive intensity thresholding. Perfusion parameter maps were calculated based on a deconvolution model by least mean squares fitting (12). Perfusion data sets with erratic or incomplete arterial and venous attenuation time curves were excluded $(n=5)$. All calculated perfusion maps were reformatted to $1 \mathrm{~mm}$ Montréal Neurological Institute (MNI) standard space using the Oxford Centre for Functional Magnetic Resonance Imaging of the Brain (FMRIB) Software Library 5.0 (Analysis Group, Oxford, $\mathrm{UK}$ ) with nearest neighbor interpolation so that quantitative image gray values remained unchanged (13). Mean voxel-wise perfusion parameter maps normalized to the MNI space were then used in our study cohort to obtain normal perfusion values for each voxel of the perfusion map and each voxel with a BM (Figures 1A-D).

\section{BM atlas}

Since CT does not offer sufficient tissue contrast for reliable detection of BM we decided to delineate and define BM location by contrast-enhanced MRI due to its superior resolution (14). One hundred consecutive patients with different tumor entities screened between $01 / 2014$ and $03 / 2015$ in our university hospital fulfilled the inclusion and exclusion criteria given in Figure 2. In particular, all patients included were not known to have BM prior to MRI and were therefore untreated for BM.

\section{MRI protocol}

The study was conducted using 1.5 Tesla MRI systems in 94 patients (Magnetom $^{\circledR}$ Sonata, Siemens Healthcare, Erlangen, Germany; Magnetom ${ }^{\circledR}$ Symphony, Siemens Healthcare, Erlangen, Germany, or Magnetom ${ }^{\circledR}$ Avanto, Siemens Healthcare, Erlangen, Germany) and 3 Tesla MRI scanners in 6 patients (Magnetom+ Skyra, Siemens Healthcare, Erlangen, Germany or Ingenia, Philips Medical Systems, Best, The Netherlands).

The standard imaging protocol always included axial T1w spin echo (2D T1 SE+) with flow compensation and/or threedimensional T1w gradient echo sequences (3D T1 GRE+) following weight-adjusted i.v. Gadolinium injection, respectively. If both sequences were acquired, axial 3D T1 GRE+ was processed for further analysis. Sequence parameters for the different MRI scanners are given in Table $\mathbf{1}$.

A necrotic BM was defined as central hypointense area surrounded by a rim of marked enhancement on post-contrast T1-weighted MR images.

\section{Segmentation of BM}

In 100 patients we detected and semi-manually segmented a total of 809 BM. Semi-automated segmentation was performed using the Analyze Software System 11.0 (Biomedical Imaging Resource, Mayo Clinic, Rochester, MN, USA) (15). Afterwards, axial post-contrast T1-weighted MR images were automatically co-registered to the MNI $1 \mathrm{~mm}$ brain by using the FMRIB

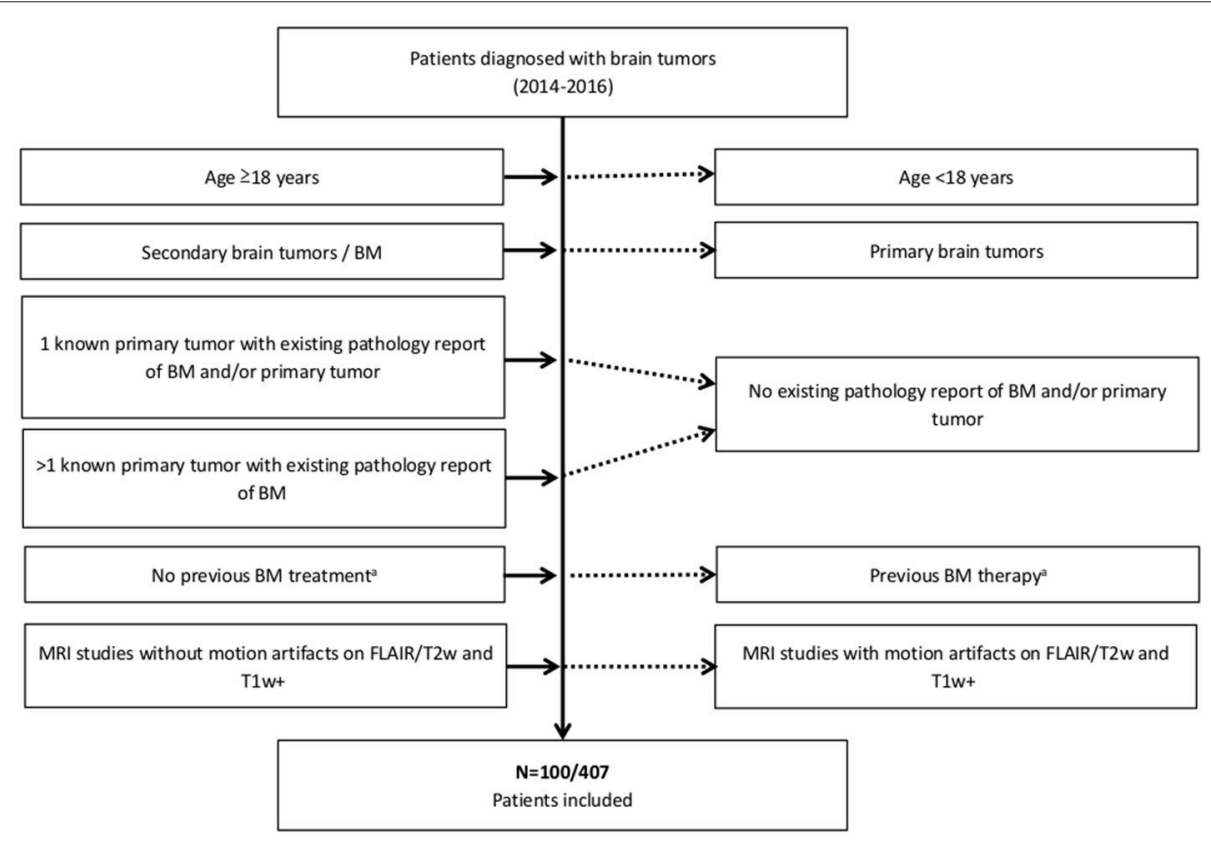

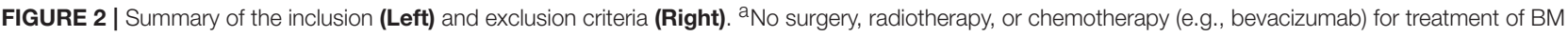
previous to $\mathrm{MRI}$. 
Software Library 5.0 linear (affine) registration tool. Correct registration of all post-contrast T1-weighted MR images and the segmented BM to the MNI brain was secured through visual inspection by two readers (T. S. and S. S.). From all BM masks in MNI space, a binary sum mask was created, subdividing the $\mathrm{MNI}$ brain in regions with $\left(\mathrm{BM}_{\mathrm{pos}}\right)$ and without $\mathrm{BM}\left(\mathrm{BM}_{\text {neg }}\right)$.

\section{Statistical Analysis}

Statistical analysis was conducted using IBM SPSS Statistics ${ }^{\circledR}$ software $\left(\mathrm{IBM}^{\circledR}\right.$ 2011, version 20, Armonk, New York, USA) and $\mathrm{R}$ (The R Foundation, version 3.3.1 Vienna, Austria). The differences in CBF, CBV, MTT, and TTP between all BMpos and $B M n e g$ voxels were compared by the independent $t$-test since we had a large enough sample size. Voxels with more than one BM were weighted according to the number of BM occurring within the voxel. Mann-Whitney $U$-test was performed to determine voxel-wise differences of perfusion values among BM with and without central necrosis. If not otherwise indicated, data are given as median \pm standard deviation.

\section{RESULTS}

\section{Descriptive Statistics}

Patient age at diagnosis of BM was $62 \pm 12.5$ years. The number of patients, number of $\mathrm{BM}$, and number of $\mathrm{BM}$ with central necrosis for the whole group and for the different primary tumors are described in Table 2. Primary tumor entities were lung cancer $(n=51: 37$ non-small lung cancer and 14 small cell lung cancer), skin cancer $(n=17: 16$ melanoma and one Merkel-cell carcinoma), breast cancer $(n=10)$, genitourinary cancer $(n=10$ : one choriocarcinoma, two prostate, two kidney, three urothelial cell cancer, and two testicular cancer), cancer of unknown primary $(n=6)$, gastrointestinal cancer ( $n=4$ : two colon and two rectal cancer), and sarcoma $(n=2)$.

\section{Perfusion Patterns}

Among all patients, CBF $(56 \pm 34$ vs. $59 \pm 35 \mathrm{ml} / 100 \mathrm{~g} / \mathrm{min}$, $p<0.001)$ and $\mathrm{CBV}(3.7 \pm 2.0$ vs. $3.9 \pm 2.1 \mathrm{ml} / 100 \mathrm{~g}, p<0.001)$ were lower and MTT $(4.8 \pm 0.7$ vs. $4.7 \pm 0.7 \mathrm{~s}, p<0.01)$ and TTP

TABLE 2 | BM characteristics among the whole cohort and the different primaries.

\begin{tabular}{lccccccccc} 
Variable & All & Lung & Skin & Breast & GU & CUP & GI & Sarcoma \\
\hline No. of patients & 100 & 51 & 17 & 10 & 10 & 6 & 4 & 2 \\
No. of BM & 809 & 312 & 199 & 146 & 42 & 47 & 41 & 22 \\
- Supratentorial & 580 & 191 & 187 & 71 & 32 & 43 & 35 & 21 \\
$\quad$ - Infratentorial & 229 & 121 & 12 & 75 & 10 & 4 & 6 & 1 \\
Necrotic changes of BM & 184 & 88 & 29 & 11 & 16 & 21 & 6 & 13
\end{tabular}

BM, brain metastasis; CUP, cancer of unknown primary; GI, gastrointestinal primary tumor; GU, genitourinary primary tumor; No., number.

TABLE 1 | Sequence parameters of the axial post-contrast T1w spin echo with flow compensation (2D T1 SE+) and/or three-dimensional T1w gradient echo sequence (3D T1 GRE+) of different MRI scanners.

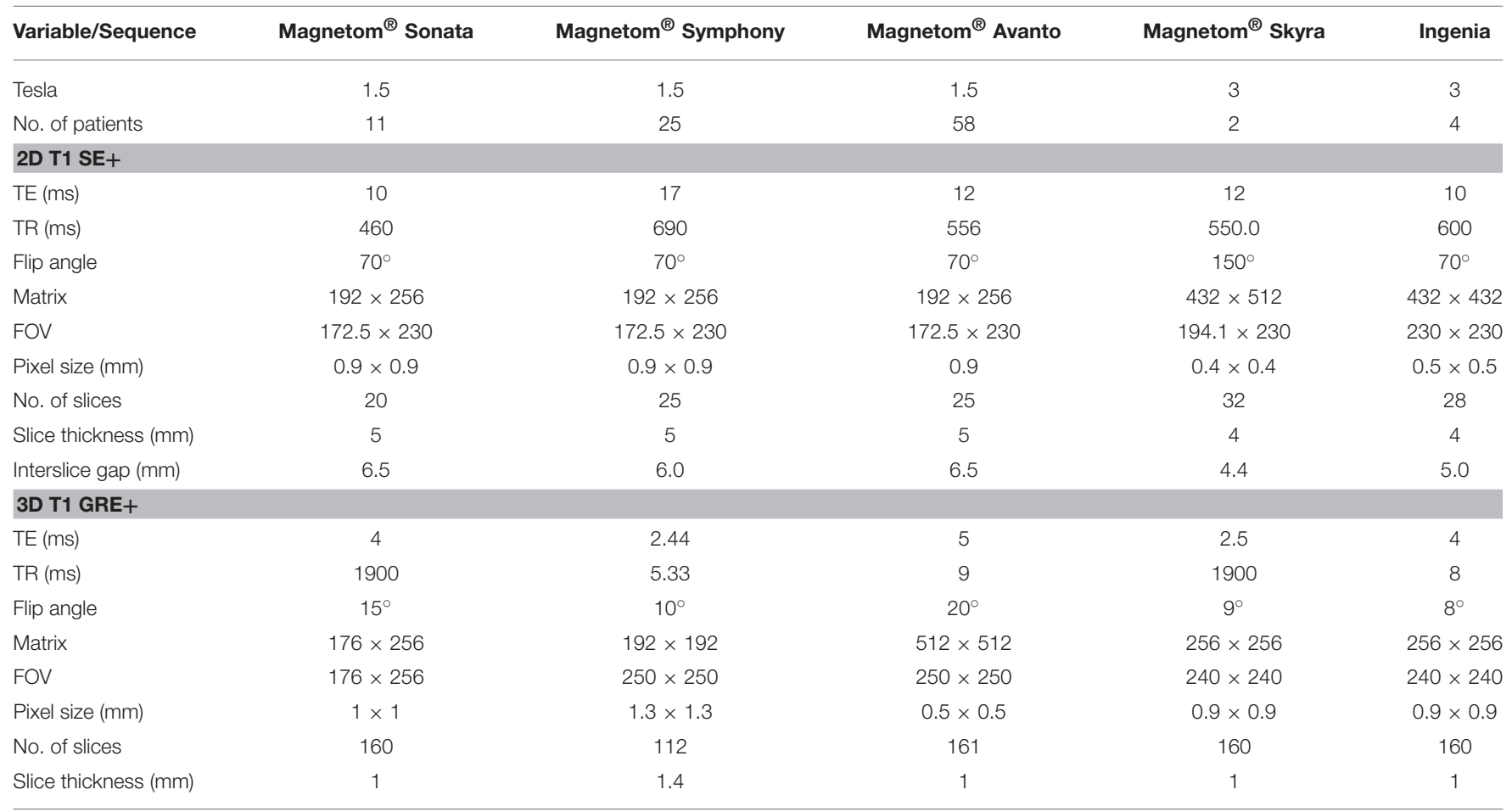

2D T1 SE+, 2-dimensional post-contrast T1-weighted spin echo with flow compensation; 3D T1 GRE+, 3-dimensional post-contrast T1-weighted gradient echo; FOV, field of view; TE, echo time; TR, repetition time. 
(9.3 \pm 0.9 vs. $9.2 \pm 0.9$ s, $p<0.001)$ were higher in $\mathrm{BM}_{\text {pos }}$ than within $\mathrm{BM}_{\text {neg. }}$.

When studying perfusion values for the supra- and infratentorial area separately, we found a marked difference: supratentorial BM occurred more often in areas with lower CBF $(51 \pm 33$ vs. $58 \pm 35 \mathrm{ml} / 100 \mathrm{~g} / \mathrm{min}, p<0.001)$ and CBV $(3.3 \pm$ 1.9 vs. $3.7 \pm 2.1 \mathrm{ml} / 100 \mathrm{~g}, p<0.001)$ and higher MTT $(4.6 \pm 0.8$ vs. $4.5 \pm 0.8 \mathrm{~s}, p<0.001)$ and TTP $(9.3 \pm 1.1$ vs. $9.1 \pm 1.1 \mathrm{~s}, p$ $<0.001)$ indicating lower perfusion compared to $\mathrm{BM}_{\text {neg. }}$. On the contrary, infratentorial BM favored areas with higher CBF (55 \pm 33 vs. $39 \pm 29 \mathrm{ml} / 100 \mathrm{~g} / \mathrm{min}, p<0.001)$ and CBV (3.7 \pm 2.0 vs. $2.7 \pm 1.9 \mathrm{ml} / 100 \mathrm{~g}, p<0.001)$ and shorter MTT $(4.7 \pm 0.7$ vs. 4.7 $\pm 0.6 \mathrm{~s}, p<0.001)$ and TTP $(9.4 \pm 0.6$ vs. $9.6 \pm 0.7 \mathrm{~s}, p<0.001)$ implying higher perfusion compared to $\mathrm{BM}_{\text {neg }}$ (Figures 3A-D).

Supratentorially, these differences were found to be greater when adjusting for the number of BM occurring within a voxel: in voxels containing a high number of $\mathrm{BM}$ the median $\mathrm{CBF}$ was even lower compared to the median $\mathrm{CBF}$ in all supratentorial voxels.

On the contrary, the highest $\mathrm{CBF}$ values infratentorially were observed in voxels containing less BM (Figures 4A,B).

\section{Perfusion and Central Necrosis}

$\mathrm{BM}$ with central necrosis showed a lower CBF ( $53 \pm 33$ vs. 59 $\pm 35 \mathrm{ml} / 100 \mathrm{~g} / \mathrm{min}, p<0.001)$ and $\mathrm{CBV}(3.5 \pm 2.0$ vs. $3.9 \pm 2.1 \mathrm{ml} / 100 \mathrm{~g}, p<0.001)$ and higher MTT $(4.8 \pm 0.8$ vs. $4.7 \pm 0.7 \mathrm{~s}, p=0.002$ ) compared to BM without necrosis. The

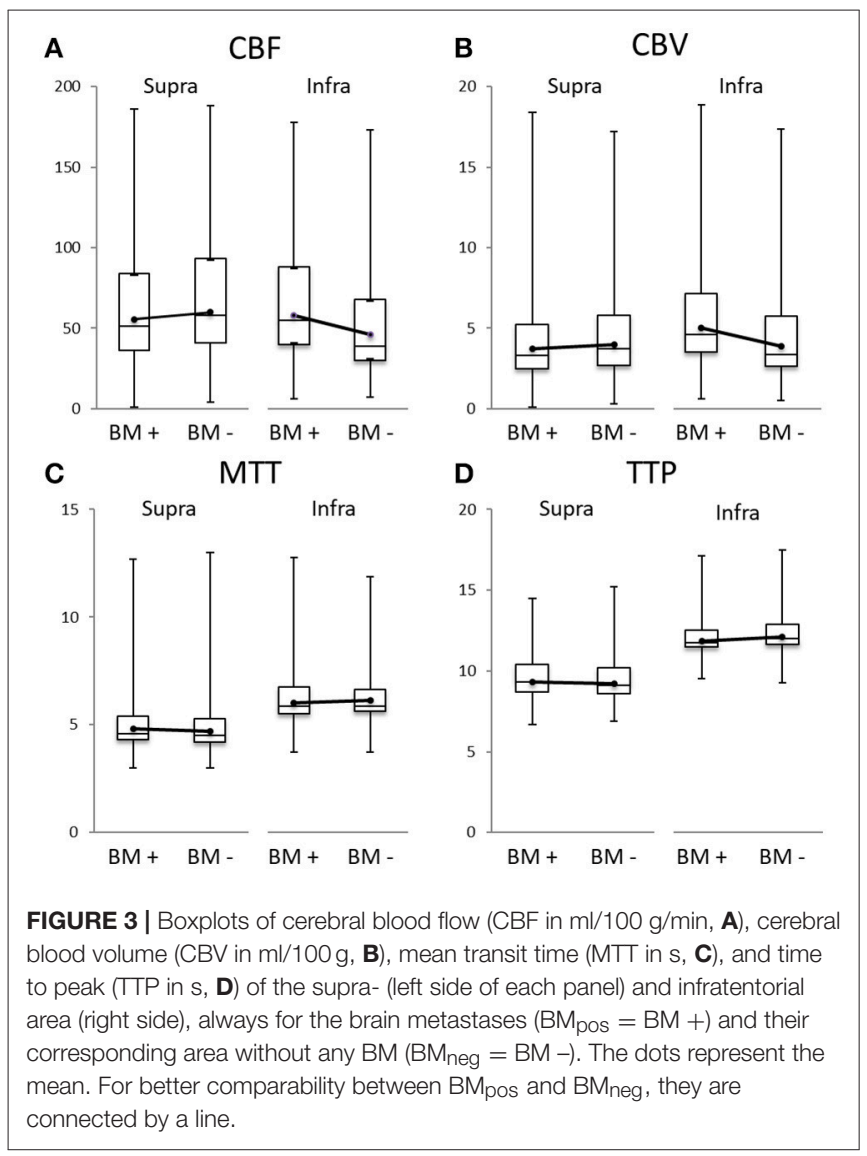

difference for TTP was not significant $(9.4 \pm 0.6$ vs. $9.3 \pm 0.6 \mathrm{~s}$, $p=0.182$ ).

\section{DISCUSSION}

In a cohort of 100 untreated $\mathrm{BM}$ patients we were able to demonstrate an inverse relationship between BM location and brain perfusion depending on lesion location. BM development in proportionally higher perfused areas occurred infratentorially whereas supratentorial BM favored lower-perfused areas. Supratentorial, these results were strengthened by our finding that the difference in perfusion increased with the number of metastases per brain voxel. Furthermore, we found that BM with central necrosis more often developed in areas with lower blood flow that corresponds to the pathophysiology of tumor necrosis.

The observed differences in BM location were greater for $\mathrm{CBF}$ and CBV and only marginal for MTT and TTP. This is likely due to the greater physiological heterogeneity of $\mathrm{CBF}$ and $\mathrm{CBV}$ between anatomical and structural brain regions whereas MTT
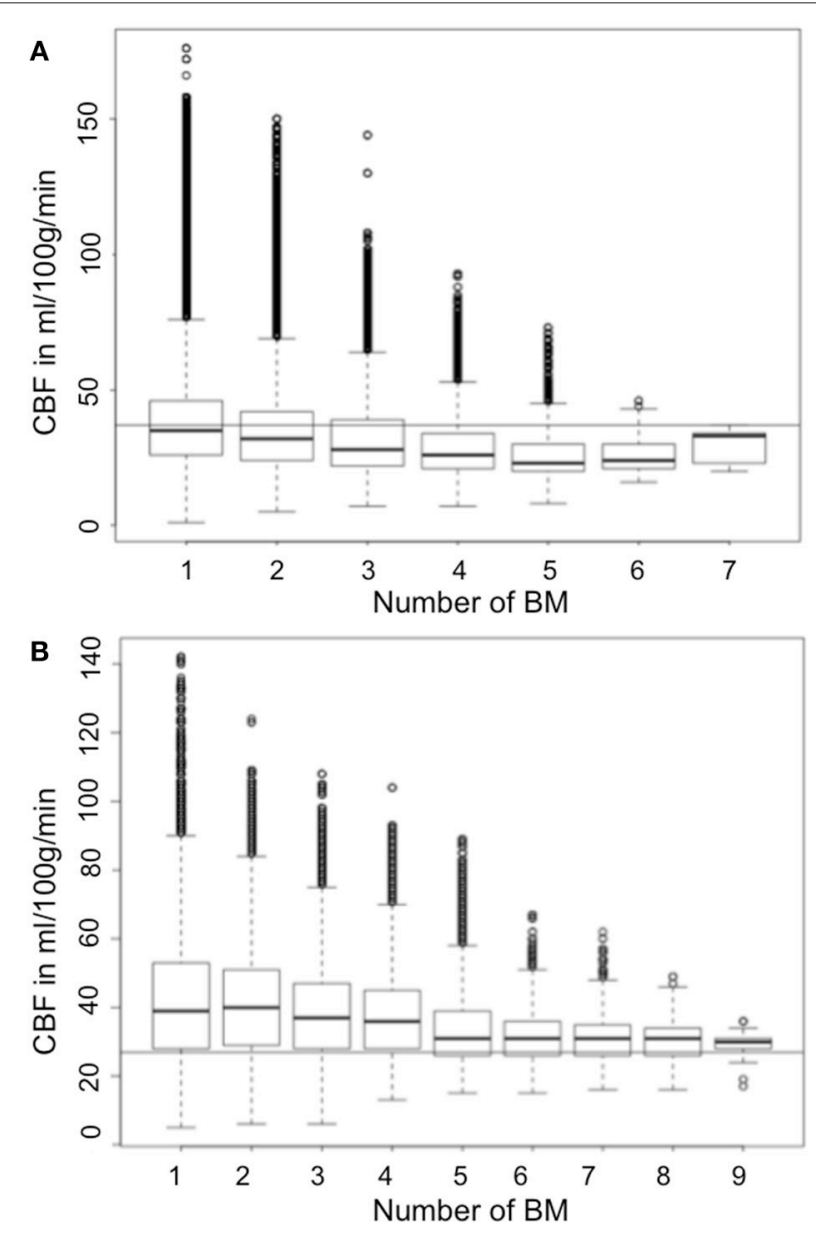

FIGURE 4 | Boxplot showing the median CBF (y-axis) according to number of $\mathrm{BM}$ ( $\mathrm{x}$-axis) in relation to the median CBF $(\mathbf{A})$ supra- and $\mathbf{( B )}$ infratentorial (continuous horizontal line). 
and TTP values of the gray and white matter are comparatively similar and do not show large variance throughout the brain (16). But what structural differences may explain our opposite supra- and infratentorial perfusion patterns? One important difference is that in evolutionary terms, the cerebellum and brainstem are older compared to the cerebral neocortex what involves a characteristic vascular architecture (17). De Reuck et al. showed that there is only one type of cerebellar cortical arterial branches whereas the vascularization of the neocortex is more complex and the type of cortical branch is cell layer-dependent (18). Furthermore, one could hypothesize that the perfusion differences are primary due to hemodynamic mechanisms. The vessel cross sectional area of the vertebral arteries is much smaller compared to the internal carotid arteries; therefore, less blood volume arrives infratentorially. Hence, higher perfusion might be a requirement for the development of cerebellar BM. We found the differences in median $\mathrm{CBF}$ and $\mathrm{CBV}$ of $\mathrm{BM}_{\text {pos }}$ and $\mathrm{BM}_{\text {neg }}$ for infratentorial BM being much greater compared to supratentorial BM what may support this hypothesis further.

Preclinical studies showed that the process of metastastic spread to the brain in lung adenocarcinoma and breast cancer cells requires production of vascular endothelial growth factor-A (VEGF-A) that induces early neoangiogenesis whereas metastatic melanoma cells were shown to proliferate along pre-existing and remodeled vessels (vessel cooption) independently from VEGF-A (19-21). Hence, our study findings could indicate that supratentorial BM rely more on VEGF-A-mediated mechanisms of angiogenesis compared to infratentorial BM.

\section{LIMITATIONS}

The main limitation of our study-since the results imply a relationship between brain perfusion and the occurrence of $\mathrm{BM}-\mathrm{is}$ that it is unclear whether the perfusion preferences also existed in the individual BM patient because it was derived from a mean perfusion map template. For this purpose, additional perfusion imaging on serial brain MRI for staging purposes could be performed in future clinical studies. However, since Nudelman et al. already demonstrated alterations of the cerebral perfusion in non-metastatic breast cancer patients, this will be difficult to interpret (9). In addition, interindividual as well as longitudinal comparison would be limited.

\section{REFERENCES}

1. Paget S. The distribution of secondary growths in cancer of the breast. 1889 . Cancer Metastasis Rev. (1989) 8:98-101.

2. Fidler IJ, Yano S, Zhang RD, Fujimaki T, Bucana CD. The seed and soil hypothesis: vascularisation and brain metastases. Lancet Oncol. (2002) 3:53-7. doi: 10.1016/S1470-2045(01)00622-2

3. Ewing J. Neoplastic Diseases: A Treatise on Tumors. 3rd ed. Philadelphia, PA: Wb saunders (1928).

4. Mazzone PJ, Marchi N, Fazio V, Taylor JM, Masaryk T, Bury L, et al. Small vessel ischemic disease of the brain and brain metastases in lung cancer patients. PLoS ONE (2009) 4:e7242. doi: 10.1371/journal.pone.0007242

5. Berk BA, Nagel S, Hering K, Paschke S, Hoffmann KT, Kortmann RD, et al. White matter lesions reduce number of brain metastases in different
Our measured perfusion parameter differences are partially very subtle (yet significant) rather for MTT/TTP than for $\mathrm{CBF} / \mathrm{CBV}$ and thus raise the question about the clinical relevance for an individual $\mathrm{BM}$ patient when observing the entire cohort. The following separation into supra- and infratentorial $\mathrm{BM}$ showed that the inverse behavior of infratentorial BM amortized initial larger perfusion value differences. Thus, we believe that our findings do have clinical significance, especially when looking for a supra- or infratentorial BM distribution. Interestingly, our median infratentorial CBV was $3.7 \mathrm{ml} / 100 \mathrm{~g}$ in $\mathrm{BM}_{\text {pos }}$ and $2.7 \mathrm{ml} / 100 \mathrm{~g}$ in $\mathrm{BM}_{\text {neg }}$ whereas the ischemia CBV threshold was reported to be $2.0 \mathrm{ml} / 100 \mathrm{~g}(16,22$, 23).

As we considered this a proof of concept study, we refrained from analyzing differences in primary tumor entities and histological or molecular subtypes. Since, for example, epidermal growth factor receptor in lung cancer was shown to influence the preferred anatomical distribution of BM, future studies should elucidate the influence of those factors on the perfusion pattern or vice versa (24).

\section{CONCLUSIONS}

We demonstrated that supratentorial BM developed preferentially in areas with low-normal perfusion, compared to $\mathrm{BM}$ of the cerebellum and brainstem, which primarily established growth in areas of high perfusion. Our findings point to diverse growth characteristics of supra- and infratentorial $B M$, which could be based on a differential vascular supply, hemodynamic requirements, production of pro-angiogenic factors, and/or anatomical homing behavior of different types of BM. Further characterization of those processes could lead to earlier detection of BM and may even provide a novel therapeutic approach.

\section{AUTHOR CONTRIBUTIONS}

Conceptualization: TS, AK, GS, and SG. Investigation: TS, AK, JS, KP, MG, GS, MM, JF, and SG. Methodology: TS, AK, GS, and SG. Software: AK and SG. Writing \pm original draft: TS, AK, JS, and SG. Writing \pm review \& editing: TS, AK, JS, KP, MG, GS, MM, JF, and SG.

cancers: a high-resolution mri study. J Neurooncol. (2016) 130:203-9. doi: 10.1007/s11060-016-2235-5

6. Quattrocchi CC, Errante Y, Mallio CA, Carideo L, Scarciolla L, Santini $\mathrm{D}$, et al. Inverse spatial distribution of brain metastases and white matter hyperintensities in advanced lung and non-lung cancer patients. J Neurooncol. (2014) 120:321-30. doi: 10.1007/s11060-014-1554-7

7. Quattrocchi CC, Errante Y, Mallio CA, Santini D, Tonini G, Zobel BB. Brain metastatic volume and white matter lesions in advanced cancer patients. $J$ Neurooncol. (2013) 113:451-8. doi: 10.1007/s11060-013-1137-z

8. Hwang TL, Close TP, Grego JM, Brannon WL, Gonzales F. Predilection of brain metastasis in gray and white matter junction and vascular border zones. Cancer (1996) 77:1551-5.

9. Nudelman KN, Wang Y, McDonald BC, Conroy SK, Smith DJ, West JD, et al. Altered cerebral blood flow one month after systemic chemotherapy for breast 
cancer: a prospective study using pulsed arterial spin labeling mri perfusion. PLoS ONE (2014) 9:e96713. doi: 10.1371/journal.pone.0096713

10. Schwarzbach CJ, Schaefer A, Ebert A, Held V, Bolognese M, Kablau $M$, et al. Stroke and cancer: the importance of cancer-associated hypercoagulation as a possible stroke etiology. Stroke (2012) 43:3029-34. doi: 10.1161/STROKEAHA.112.658625

11. Feyen L SH, Niederstadt T, Heindel W, Kemmling A Standardization of dynamic whole brain perfusion ct: a comprehensive database of regional perfusion parameters; dgnr 2010 - jahrestagung der deutschen gesellschaft für neuroradiologie. Clin Neuroradiol. (2010) 20:189-220. doi: 10.1007/s00062-010-0025-6

12. Abels B, Klotz E, Tomandl BF, Kloska SP, Lell MM. Perfusion ct in acute ischemic stroke: a qualitative and quantitative comparison of deconvolution and maximum slope approach. AJNR Am J Neuroradiol. (2010) 31:1690-98. doi: 10.3174/ajnr.A2151

13. Kemmling A, Wersching H, Berger K, Knecht S, Groden C, Nolte I. Decomposing the hounsfield unit: Probabilistic segmentation of brain tissue in computed tomography. Clin Neuroradiol. (2012) 22:79-91. doi: 10.1007/s00062-011-0123-0

14. Sze G, Milano E, Johnson C, Heier L. Detection of brain metastases: comparison of contrast-enhanced $\mathrm{mr}$ with unenhanced $\mathrm{mr}$ and enhanced ct. AJNR Am J Neuroradiol. (1990) 11:785-91.

15. Robb RA. The biomedical imaging resource at mayo clinic. IEEE Trans Med Imaging (2001) 20:854-67. doi: 10.1109/42.952724

16. Konstas AA, Goldmakher GV, Lee TY, Lev MH. Theoretic basis and technical implementations of ct perfusion in acute ischemic stroke, part 2: technical implementations. AJNR Am J Neuroradiol. (2009) 30:885-92. doi: 10.3174/ajnr.A1492

17. Florio M, Huttner WB. Neural progenitors, neurogenesis and the evolution of the neocortex. Development (2014) 141:2182-94. doi: 10.1242/dev.090571

18. De Reuck J. The cortico-subcortical arterial angio-architecture in the human brain. Acta Neurol Belg. (1972) 72:323-9.

19. Kienast $Y$, von Baumgarten L, Fuhrmann M, Klinkert WE, Goldbrunner R, Herms J, et al. Real-time imaging reveals the single steps of brain metastasis formation. Nat Med. (2010) 16:116-22. doi: 10.1038/nm.2072
20. Yano S, Shinohara H, Herbst RS, Kuniyasu H, Bucana CD, Ellis LM, et al. Expression of vascular endothelial growth factor is necessary but not sufficient for production and growth of brain metastasis. Cancer Res. (2000) 60:4959-67.

21. Kim LS, Huang S, Lu W, Lev DC, Price JE. Vascular endothelial growth factor expression promotes the growth of breast cancer brain metastases in nude mice. Clin Exp Metastasis (2004) 21:107-18. doi: 10.1023/B:CLIN.0000024761.003 73.55

22. Wintermark M, Flanders AE, Velthuis B, Meuli R, van Leeuwen $\mathrm{M}$, Goldsher D, et al. Perfusion-ct assessment of infarct core and penumbra: receiver operating characteristic curve analysis in 130 patients suspected of acute hemispheric stroke. Stroke (2006) 37:979-85. doi: 10.1161/01.STR.0000209238.614 59.39

23. Wintermark M, Reichhart M, Thiran JP, Maeder P, Chalaron M, Schnyder $\mathrm{P}$, et al. Prognostic accuracy of cerebral blood flow measurement by perfusion computed tomography, at the time of emergency room admission, in acute stroke patients. Ann Neurol. (2002) 51:417-32. doi: 10.1002/ana. 10136

24. Takano K, Kinoshita M, Takagaki M, Sakai M, Tateishi S, Achiha T, et al. Different spatial distributions of brain metastases from lung cancer by histological subtype and mutation status of epidermal growth factor receptor. Neuro Oncol. (2016) 18:716-24. doi: 10.1093/neuonc/nov266

Conflict of Interest Statement: The authors declare that the research was conducted in the absence of any commercial or financial relationships that could be construed as a potential conflict of interest.

Copyright (C) 2018 Schneider, Kemmling, Schroeder, Pantel, Glatzel, Schoen, Mohme, Fiehler and Gellißen. This is an open-access article distributed under the terms of the Creative Commons Attribution License (CC BY). The use, distribution or reproduction in other forums is permitted, provided the original author(s) and the copyright owner are credited and that the original publication in this journal is cited, in accordance with accepted academic practice. No use, distribution or reproduction is permitted which does not comply with these terms. 